\title{
Original article (full paper) \\ Effects of water ingestion throughout exercise and recovery on cardiac autonomic modulation during and after exercise
}

\author{
Isadora Lessa Moreno \\ Universidade Federal de São Paulo, São Paulo, SP, Brasil \\ Luiz Carlos Marques Vanderlei \\ Franciele Marques Vanderlei \\ Carlos Marcelo Pastre \\ Universidade Estadual Paulista “Julio de Mesquita Filho”, Presidente Prudente, SP, Brasil \\ Luiz Carlos de Abreu \\ Faculdade de Medicina do ABC, Santo André, SP, Brasil \\ Celso Ferreira \\ Universidade Federal de São Paulo, São Paulo, SP, Brasil
}

\begin{abstract}
Purpose: To analyze the effects of water intake on heart rate variability (HRV) during and after exercise. Methods: Thirty-one young males performed three different procedures: I) a maximal exercise test to determine the load for the protocols; II) the control protocol (CP); III) the experimental protocol (EP). The protocols comprised 10 minutes at rest, 90 minutes of treadmill exercise $\left(60 \%\right.$ of $\mathrm{VO}_{2}$ peak), and 60 minutes of recovery. No rehydration beverage consumption was allowed during the CP. During the EP, the participants were given water every 15 minutes, being that the amount of water to be ingested by each volunteer was divided over the time of exercise and recovery. For the analysis of HRV data, time and frequency domain indices were investigated in the following moments (M): M1 (5 minutes final of rest), M2 (25-30 minutes of exercise), M3 (55-60 minutes of exercise), M4 (85-90 minutes of exercise), M5 (5-10 minutes of recovery), M6 (15-20 minutes of recovery), M7 (25-30 minutes of recovery), M8 (40-45 minutes of recovery), and M9 (55-60 minutes of recovery). Results: There was no statistically significant difference between the groups that were examined during the exercise. HRV values for the CP and EP at the end of the exercise period were as follows SDNN (5.95 \pm 1.89 vs $6.89 \pm 2.11)$; RMSSD (3.29 \pm 0.78 vs $3.39 \pm 0.95)$; HF [nu] (24.06 \pm 14.26 vs $19.10 \pm 11.94) ; \mathrm{LF}$ [nu] ( $75.93 \pm 14.26$ vs $80.89 \pm 11.94) ; \mathrm{HF}\left[\mathrm{ms}^{2}\right](0.90 \pm 1.16$ vs $0.94 \pm 0.89)$; LF [ms $\left.{ }^{2}\right]$ (4.65 \pm 5.94 vs $5.35 \pm 5.09)$; and $\mathrm{LF} / \mathrm{HF}$ ratio $(4.64 \pm 3.15$ vs $6.95 \pm 5.57)$. HRV values for the $\mathrm{CP}$ and the $\mathrm{EP}$ at the end of the recovery period were as follows: SDNN ( $39.63 \pm 19.63$ vs $49.95 \pm 17.79)$; RMSSD (21.63 \pm 15.55 vs $36.43 \pm 22.73)$; HF $\left[\mathrm{ms}^{2}\right]$ (285.51 \pm 392.27 vs 699.29 \pm 699.66$)$; LF [nu] (74.55 \pm 13.25 vs $\left.61.19 \pm 19.27\right)$; and HF [nu] (25.44 \pm 13.25 vs 38.8 \pm 19.27$)$. Conclusion: The hydration with water was not enough to significantly influence the linear HRV indices during exercise; however, it promoted faster recovery of these variables after exercise.
\end{abstract}

Keywords: exercise, rehydration solutions, autonomic nervous system, heart rate

\section{Introduction}

The process of dehydration occurs when fluid loss is not compensated by the ingestion of fluid, which in turn will lead to a deterioration of temperature regulation, performance, and health. Ensuring adequate fluid intake before, during, and after exercise can improve performance and reduce the problems associated with heat ${ }^{1-4}$.

Efficient hydration ensures the ideal conditions for the practitioner to maintain their physical capability to provide proper functioning of the homeostatic processes required by exercise ${ }^{5}$.

From a physiological standpoint, studies have shown the benefits of water intake during and/or after exercise. A previous study subjected ten individuals to ingestion of water immediately after a 30 -minute cycling exercise at a workload representing $80 \%$ of anaerobic threshold. They showed that water consumption accelerated cardiac vagal reactivation after exercise; however, no significant influence on heart rate (HR) and blood pressure responses was observed ${ }^{6}$. In another study, the participants were subjected to a protocol of hydration during and after exercise: the results showed minimal changes in systolic blood pressure (SBP) and diastolic blood pressure (DBP) and a smaller increase in HR during exercise ${ }^{7}$. The results showed better HR recovery and faster return of SBP and DBP and better performance of pulse oxygen saturation and respiratory rate. Peçanha, Paula-Ribeiro, Campana-Rezende, 
Bartels, Marins, de Lima ${ }^{8}$ evaluated the effects of water intake on the parasympathetic recovery after high-intensity exercise in active young individuals and found that water accelerates post-exercise vagal reactivation.

Although studies have shown that water intake (the method of hydration most commonly used) before or during exercise is beneficial to cardiac autonomic responses post-exercise, it is not known what is the effect of water intake performed repeatedly throughout the exercise and recovery period on these responses. In addition, the studies did not evaluate these responses for an extended recovery time. Therefore, our purpose was to evaluate the effects of hydration protocols on autonomic modulation of the heart in young people during and post-exercise. We hypothesized that hydration with water during exercise and recovery may attenuate autonomic changes induced by exercise and accelerate recovery. To test this hypothesis, we assessed linear indices of heart rate variability (HRV) in young men with and without water intake (Vittalev, Spaipa, Brazil).

\section{Methods}

\section{Participants}

Thirty-one healthy, young male volunteers $(21.5 \pm 1.8 \mathrm{yrs})$ were investigated. All were active according to the International Physical Activity Questionnaire (IPAQ) ${ }^{9}$. These volunteers had no damaging habits such as smoking or alcohol consumption, were not taking medications that influence cardiac autonomic activity, and were not diagnosed with any cardiovascular, metabolic or endocrine disorder. No volunteers were excluded during the course of the experiment. Every individual signed a consent letter and was informed of the procedures and objectives of the study. The study's procedures were all approved by the Research Ethics Committee of the Federal University of São Paulo-UNIFESP (Number 0861/11).

\section{Experimental design protocol}

For all visits, volunteers were instructed to avoid consuming caffeine $24 \mathrm{~h}$ before the procedures, to consume a light fruitbased meal $2 \mathrm{~h}$ before the tests, to have a good night's sleep (7-8 h), to avoid strenuous physical exercise the day before the test and to wear appropriate and comfortable clothes (shorts, shirt, shoes, and socks) for physical exercise.

The clinical trial was based on previous studies ${ }^{10,11}$. Participants reported to the laboratory three days per week, at an interval of 48 hours between visits. An incremental test was applied during the first visit, which was performed on a treadmill (Super ATL, Inbrasport, Brazil) according to the Bruce proto$\mathrm{col}^{12}$. To establish the baseline, volunteers were allowed to rest in a standing position on the mat before the test began. Once the test started, verbal encouragement was used in an attempt to obtain a maximum physical effort; the test was interrupted by voluntary exhaustion. To determine oxygen consumption $\left(\mathrm{VO}_{2}\right)$, expired gases were analyzed using a regularly calibrated metabolic analyzer (VO2000, Medical Graphics, St Paul, MN, USA). The $\mathrm{VO}_{2}$ peak was taken to be the highest $\mathrm{VO}_{2}$ achieved in the test. The HR reached at $60 \%$ of $\mathrm{VO}_{2}$ peak was used to determine the exercise intensity for the protocols, considering that gastric emptying is considerably disturbed at intensities above $70 \%$ of $\mathrm{VO}_{2}$ peak $^{13}$.

In subsequent visits, called control (CP) and experimental (EP) protocols, volunteers were allowed to rest in the supine position for 10 minutes, followed by 90 minutes of exercise (60\% of $\mathrm{VO}_{2}$ peak), and 60 minutes of recovery. Volunteers were not given any fluids to drink during the CP; however, during the EP they were given water (Vittalev, Spaipa, Brazil) containing bicarbonate $(62.49 \mathrm{mg} / \mathrm{L})$, calcium $(7.792 \mathrm{mg} / \mathrm{L})$, carbonate $(3.91 \mathrm{mg} / \mathrm{L})$, chloride $(0.09 \mathrm{mg} / \mathrm{L})$, strontium $(0.343$ $\mathrm{mg} / \mathrm{L})$, fluorid $(0.05 \mathrm{mg} / \mathrm{L})$, magnesium $(0.340 \mathrm{mg} / \mathrm{L})$, potassium (1.485 mg/L), sodium (16.090 mg/L), and sulfate $(0.18 \mathrm{mg} / \mathrm{L})$. Water intake was administered in 10 equal portions at regular intervals of 15 minutes from the fifteenth minute of exercise until the end of the recovery. They were given water at temperatures between 15 and $22^{\circ} \mathrm{C}$. The amount of water administered was based on the difference in body weight before and after the CP, therefore the $\mathrm{CP}$ was always realized before the $\mathrm{EP}$ - there was no randomization. This technique indicates that $1 \mathrm{~g}$ reduction in body weight is equal to $1 \mathrm{~mL}$ of fluid reduction ${ }^{14}$.

The protocols were performed in a room under environment temperatures $\left(26.0 \pm 2.3^{\circ} \mathrm{C}\right)$ and humidity $(55.1 \pm 10.4 \%)$ between 3 p.m. and 6 p.m. to avoid circadian variations. To ensure the condition of initial hydration all the volunteers drank water $(500 \mathrm{~mL})$ at one time $2 \mathrm{~h}$ before both protocols ${ }^{1}$. Body weight (digital scale Plenna, TIN 00139 MÁXIMA, Brazil), and height (stadiometer ES 2020, Sanny, Brazil) were measured in all the subjects upon their arrival at the laboratory. The heart monitor was then strapped on each subject's thorax over the distal third of the sternum. The HR receiver (Polar Electro S810i, Kempele, Finland) was placed on the wrist for beat-to-beat HR measurements and for HRV analysis.

HR was analyzed at the following periods: final 10 minutes of rest; after 30, 60, and 90 minutes of exercise; after 5, 10, 20, $30,40,50$, and 60 minutes of recovery.

The volunteers remained at rest in the supine position for 10 minutes and immediately their axillary temperature (thermometer BD Thermofácil, China) was measured. Subsequently, the participants performed a treadmill exercise $\left(60 \%\right.$ of $\mathrm{VO}_{2}$ peak) for 90 minutes and were then allowed to rest in the supine position for 60 minutes for recovery. Axillary temperature was checked again immediately following exercise; the volunteers' nude weight was measured again at the end of the recovery period.

Urine was collected and analyzed (10 Choiceline, Roche $\AA$, Brazil) at the end of the EP and after measurement of final body weight. Urine density was used as a marker for hydration level ${ }^{15}$.

\section{Heart rate variability indices analysis}

HRV was recorded beat-to-beat through the monitoring process (Polar Electro S810i) at a sampling rate of $1000 \mathrm{~Hz}$. During the period of higher signal stability, verified of visual 
form, an interval of 5 minutes was selected, and series with more than $256 \mathrm{RR}$ intervals were used for analysis, following digital filtering complemented by manual filtering to eliminate premature ectopic beats and artifacts. Only series with more than $95 \%$ sinus rhythm were included in the study ${ }^{16,17}$. To analyze $\mathrm{HRV}$ in the frequency domain, we used the low (LF) and high frequencies (HF) spectral components in normalized units (nu) and absolute $\left(\mathrm{ms}^{2}\right)$, and the LF/HF ratio, which represents the relationship between low and high frequencies ${ }^{16}$. We considered the following range: $\mathrm{LF}, 0.04-0.15 \mathrm{~Hz}$ and $\mathrm{HF}, 0.15-0.4 \mathrm{~Hz}$. The spectral analysis was calculated using the Fast Fourier Transform algorithm ${ }^{18}$. Analysis in the time domain was performed by means of SDNN (ms) (standard deviation of normal-to-normal RR intervals) and RMSSD (ms) (root-mean square of differences between adjacent normal RR intervals in a time interval $)^{16}$.

HRV indices were analyzed at the following moments: M1 (final 5 minutes rest); M2 (25-30 minutes after exercise); M3 (55-60 minutes after exercise); M4 (85-90 minutes after exercise); M5 (5-10 minutes of recovery); M6 (15-20 minute recovery); M7 (25-30 minute recovery); M8 (40-45 minute recovery) and M9 (55-60 minute recovery). Series with more than 256 RR intervals were used for analysis ${ }^{16}$. We used Kubios HRV version 2.0 software to analyze these indices ${ }^{19}$.

\section{Statistical Analysis}

Gaussian distribution of the data was verified using the Shapiro-Wilks test. For comparisons between protocols (control vs. experimental) and moments (M1, M2, M3 and M4 during exercise and M1 vs. M5, M6, M7, M8, M9 during recovery) two-way repeated measures analysis of variance was applied, followed by the Bonferroni post-test for parametric distributions or Dunn's post-test for non-parametric data. The repeated measures data were checked for sphericity violation using Mauchly's test and the Greenhouse-Geisser correction was conducted when sphericity was violated. Significance level was set at $p<0.05$ for all tests. SPSS (version 13.0) software (SPSS Inc., Chicago, IL, USA) was used for statistical analysis.

The sample size calculation was done considering how variable the RMSSD index. The magnitude of assumed significant difference was $12 \mathrm{~ms}$, considering a standard deviation of 16 $\mathrm{ms}$, with alpha risk of $5 \%$ and beta of $80 \%$, and the sample size resulted in 28 young. Considering possible sample loss, adds up $10 \%$ to sample size calculated, totaling 31 young.

\section{Results}

The anthropometric characteristics of the participants and their responses obtained during the incremental test are described in Table 1. Table 2 shows data regarding body mass and temperature in the $\mathrm{CP}$ and the $\mathrm{EP}$. We observed weight loss in the $\mathrm{CP}$ (Table 2). The percentage of body weight loss in the CP was $2.0 \pm 0.6 \%$, while in the EP it was $0.1 \pm 0.8 \%$. The average consumption of water was $1.4 \pm 0.5 \mathrm{~L}$ in the EP. The density of urine $(1.017 \pm 0.004 \mathrm{~g} / \mathrm{mL})$ evaluated at the end of the EP confirms that the volume of solution intake was sufficient to maintain the participants at euhydrated status ${ }^{15}$. Body temperature behaved similarly in both protocols, increasing significantly at the end of the exercise (Table 2).

Table 1. Subject characteristics.

\begin{tabular}{|c|c|c|}
\hline Variables & $\begin{array}{c}\text { Mean } \pm \\
\text { Standard Deviation }\end{array}$ & $\begin{array}{l}\text { Minimum/ } \\
\text { Maximum }\end{array}$ \\
\hline \multicolumn{3}{|l|}{ Anthropometric } \\
\hline data & $21.5 \pm 1.8$ & {$[18-25]$} \\
\hline Age (yr) & $72.6 \pm 11.5$ & {$[53.8-95.3]$} \\
\hline Body mass (kg) & $1.70 \pm 0.10$ & {$[1.60-1.90]$} \\
\hline Height (m) & $23.0 \pm 2.8$ & {$[16.8-28.1]$} \\
\hline \multicolumn{3}{|l|}{$\operatorname{BMI}\left(\mathrm{kg} / \mathrm{m}^{2}\right)$} \\
\hline Incremental test & $3.3 \pm 0.6$ & {$[2.0-5.1]$} \\
\hline $\mathrm{VO}_{2 \text { peak }}\left(\mathrm{L} \cdot \mathrm{min}^{-1}\right)$ & $160.7 \pm 10.7$ & {$[139-179]$} \\
\hline \multicolumn{3}{|l|}{$\mathrm{HR}(\mathrm{bpm})$ at $60 \%$} \\
\hline \multicolumn{3}{|l|}{$\mathrm{VO}_{2 \text { peak }}$} \\
\hline $\begin{array}{c}\mathrm{VO}_{2 \text { peak }} \text { relative to } \\
\text { body mass } \\
\left(\mathrm{L} \cdot \mathrm{min}^{-1} / \mathrm{Kg}\right)\end{array}$ & $0.047 \pm 0.007$ & {$[0.027-0.058]$} \\
\hline
\end{tabular}

Legend: yr, years; kg, kilograms; m, meters; BMI, body mass index; VO2peak, peak oxygen consumption; HR, heart rate; bpm, beats per minute; L.min-1, liter per minute.

Table 2. Values of body mass and temperature in control and experimental protocols.

\begin{tabular}{cccc}
\hline Variable & Moment & $\begin{array}{c}\text { Control protocol } \\
\text { Mean } \pm \text { Standard Deviation } \\
\text { Median [minimum-maximum] }\end{array}$ \\
\hline Body mass $(\mathrm{kg})$ & $\begin{array}{c}\text { Experimental } \\
\text { protocol }\end{array}$ \\
& protocol & $\begin{array}{c}73.0 \pm 11.6 * \\
(72.9)\end{array}$ & $\begin{array}{c}73.1 \pm 11.4 \\
(73.2)\end{array}$ \\
& & {$[54.7-96.1]$} & {$[53.5-96.6]$} \\
& After the & $71.6 \pm 11.3$ & $73.0 \pm 11.5$ \\
& protocol & $(71.0)$ & $(72.6)$ \\
& & {$[53.6-94.2]$} & {$[53.5-97]$} \\
& $\Delta(\%)$ & $2.0 \pm 0.6$ & $0.1 \pm 0.8$ \\
Body & Before & $36.4 \pm 0.5 \#$ & $36.5 \pm 0.4 *$ \\
temperature $\left({ }^{\circ} \mathrm{C}\right)$ & exercise & $(36.5)$ & $(36.5)$ \\
& & {$[35-38]$} & {$[35-36.9]$} \\
& After & $37.2 \pm 0.5$ & $37.0 \pm 0.5$ \\
& & $(37.2)$ & $(37.0)$ \\
& & {$[35.5-38]$} & {$[36.0-38.0]$} \\
\hline
\end{tabular}

Legend: $\mathrm{kg}$, kilogram; ${ }^{\circ} \mathrm{C}$, degrees Celsius; $\Delta$, delta; \%, percentage. *Value with a significant difference compared with after the exercise (Student $t$ test of paired data; $\mathrm{p}<.05$ ); \#value with a significant difference compared with after the exercise (Wilcoxon test; $\mathrm{p}<.05$ );

Figure 1 shows HR values during exercise (Figure 1a) and recovery (Figure 1b). During exercise, we observed the effect of moment $(p<0.001)$ on HR; however, there was no effect among protocols $(p=0.31)$ and in the moment and protocol interaction $(p=0.29)$. In both protocols, we noted that HR was significantly increased at 30,60, and 90 minutes of exercise compared to rest, however, although not significant, the increase was lower in the EP. In the recovery period, we observed the effects of moment $(p<0.001)$, and moment and protocol interaction $(p=0.006)$ 
on HR; there was no effect among protocols $(p=0.081)$. In the $\mathrm{CP}$, a significant decrease was observed when comparing all minutes of recovery at rest, while in the EP it was only observed in the $1,3,5,7,10$, and 30 minute. In the EP, after 40 minutes of recovery the HR did return to baseline.

Figures 2 and 3 show the behavior of HRV indices in time and frequency domains, respectively, during exercise. There was a moment effect for the time domain indices (SDNN and RMSSD; $p$ $<0.001)$. No effects were observed between the protocols (SDNN, $p=0.66$; RMSSD, $p=0.94)$ and in the moment and protocol interaction (SDNN, $p=0.91$; RMSSD, $p=0.88$ ). We noted that SDNN (ms) and RMSSD (ms) were significantly decreased at M2, M3, and M4 of exercise in both the CP and EP compared to M1 (rest). Moreover, there was a decrease in the SDNN (ms) for the CP at M2 of exercise compared to M4 of exercise.
Likewise, we observed a moment effect in all indices in the frequency domain $(p<0.001)$. No effects were observed for those indices between the protocols [LF $\left(\mathrm{ms}^{2}\right), p=0.50$; $\mathrm{HF}\left(\mathrm{ms}^{2}\right), p=0.72 ; \mathrm{LF}(\mathrm{nu}), p=0.63$; HF (nu), $p=0.63 ; \mathrm{LF} /$ $\mathrm{HF}, p=0.31]$. There were no interactions between moment and protocol $\left[\mathrm{LF}\left(\mathrm{ms}^{2}\right), p=0.53 ; \mathrm{HF}\left(\mathrm{ms}^{2}\right), p=0.71, \mathrm{LF}(\mathrm{nu})\right.$, $p=0.64$; HF (nu), $p=0.64, \mathrm{LF} / \mathrm{HF}, p=0.87]$. Regarding the comparison between moments, we observed that LF $\left(\mathrm{ms}^{2}\right)$, $\mathrm{HF}\left(\mathrm{ms}^{2}\right)$, and HF (nu) were significantly higher at M1 (rest) compared to M2, M3, and M4 of exercise in both the CP and the EP. LF (nu) and LF/HF were significantly lower at M1 compared to M2, M3 and M4 of exercise in both the CP and the EP. Moreover, LF $\left(\mathrm{ms}^{2}\right)$ was significantly higher at M2 of exercise compared to M4 of exercise in the CP, while HF $\left(\mathrm{ms}^{2}\right)$ was significantly higher at M2 of exercise compared to M4 of exercise in the EP.
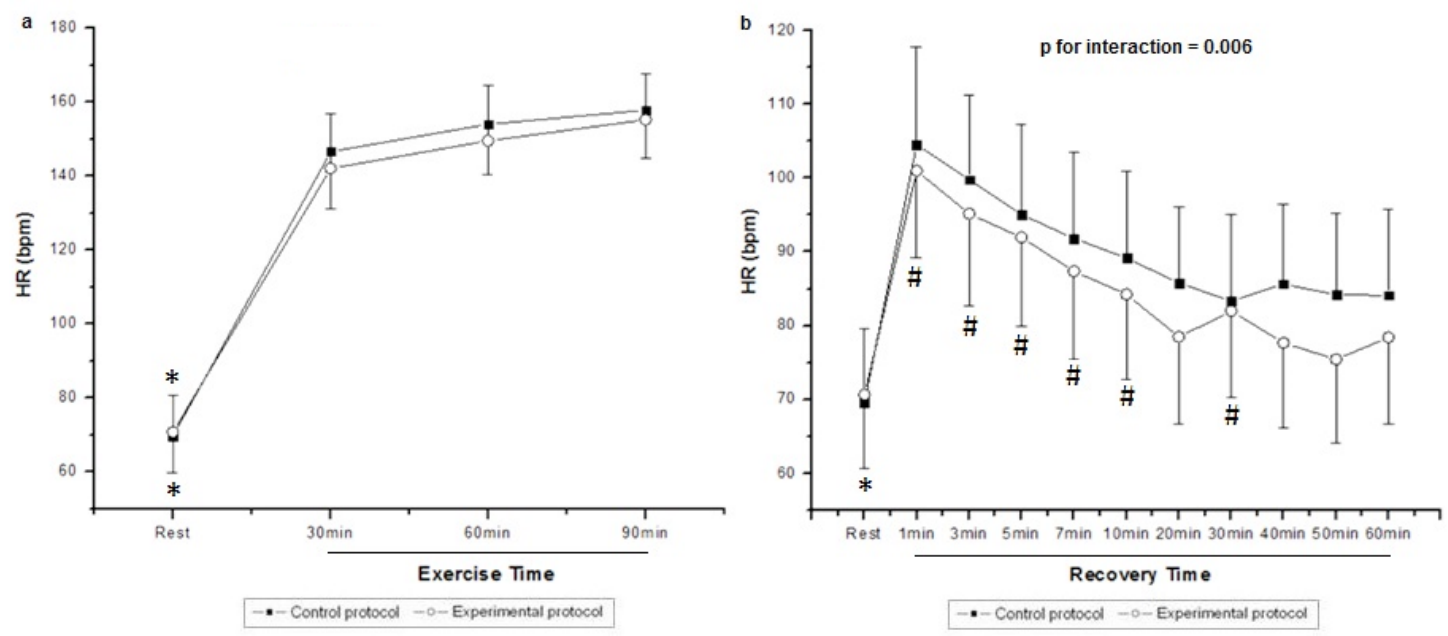

Figure 1. Values are mean \pm standard deviation. Heart rate (HR) during (a) exercise and (b) recovery, and the comparison in control and experimental protocols; *Different from all the times of exercise and recovery $(\mathrm{p}<0.05)$; \#Different from M1 $(\mathrm{p}<0.05)$; HR, heart rate; bpm, beats per minute.
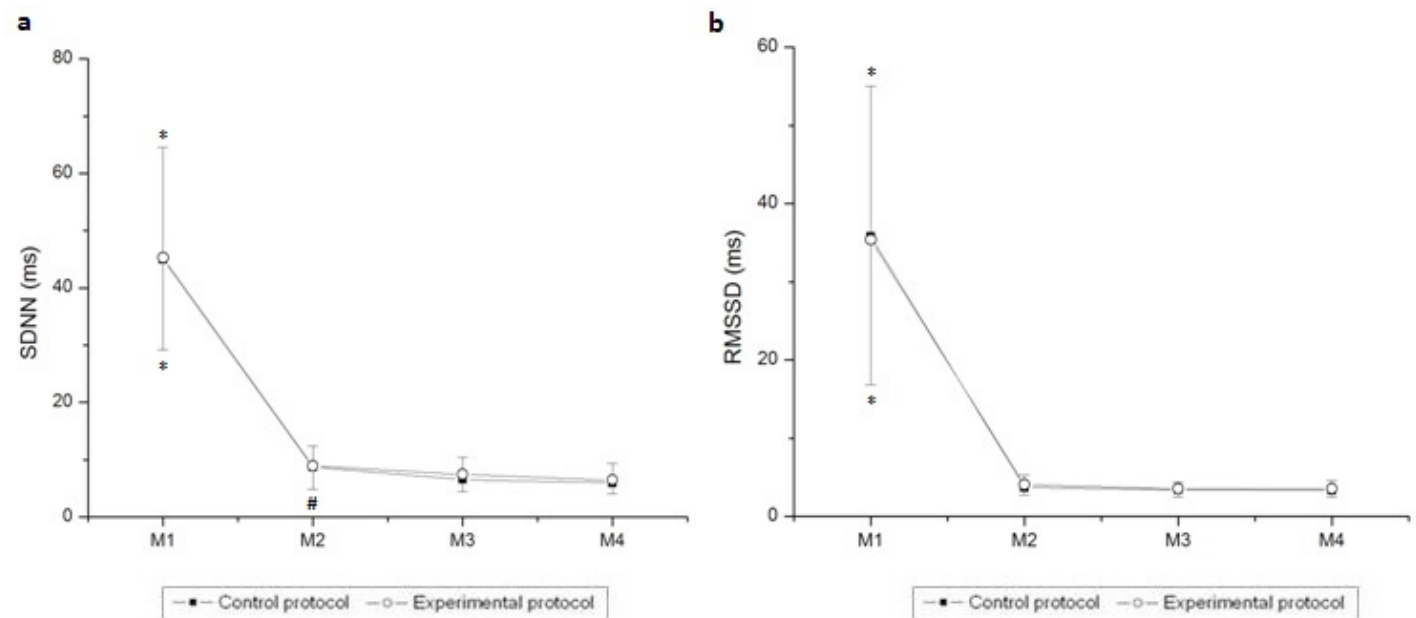

Figure 2. Values are mean \pm standard deviation. (a) SDNN and (b) RMSSD during exercise and the comparison in control and experimental protocols. Final 5 minutes of rest (M1) and minutes of exercise: 25-30 (M2), 55-60 (M3), 85-90 (M4). *Different from M2, M3, and M4 (p < 0.05). \#Different from M4 in the CP ( $\mathrm{p}<0.05)$; SDNN, standard deviation of the mean of all normal RR intervals; RMSSD, square root of the mean squared differences between successive normal RR intervals; $\mathrm{M}$, moment; ms, milliseconds. 

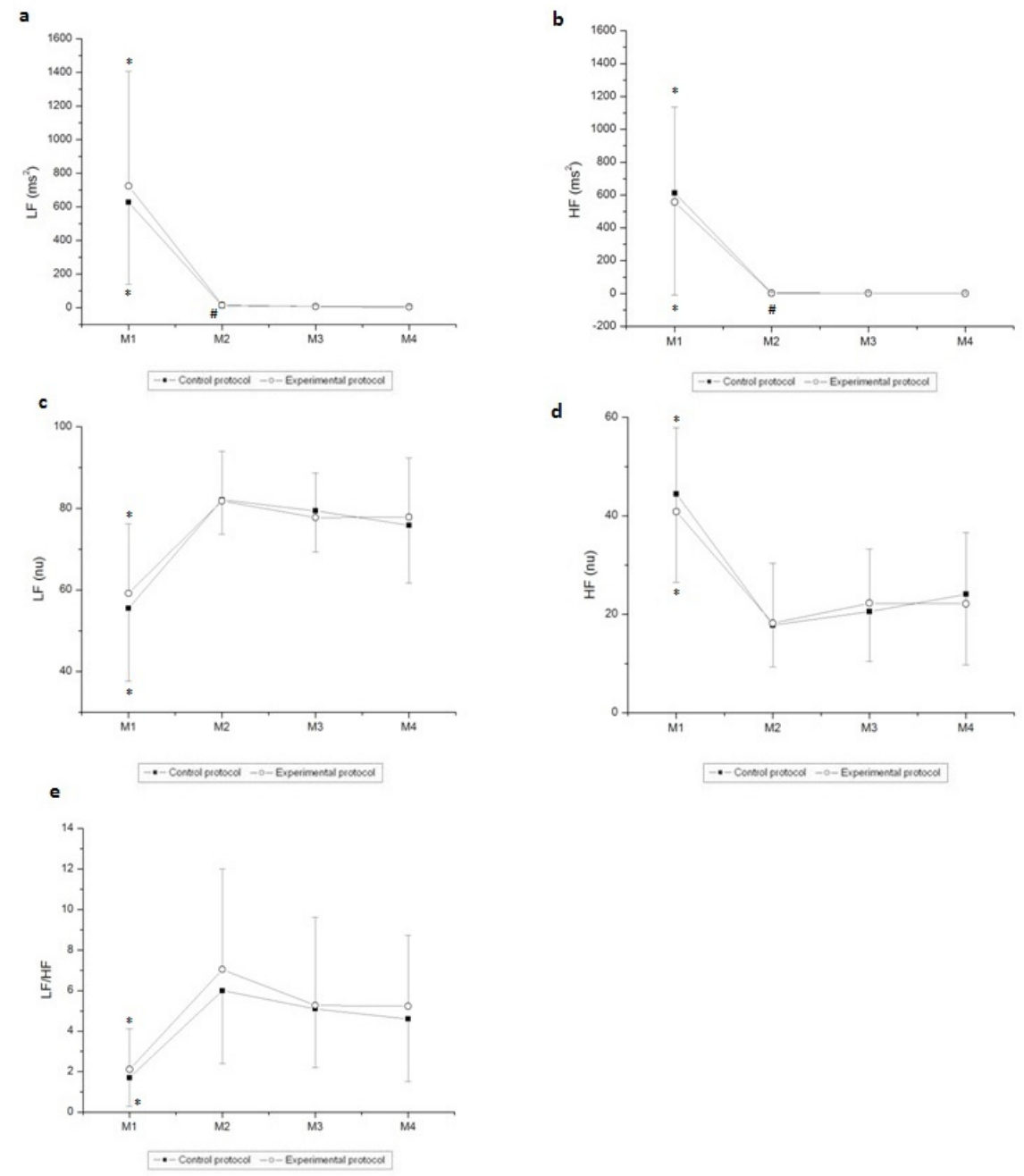

Figure 3. Values are mean \pm standard deviation. (a) $\mathrm{LFms}^{2}$, (b) $\mathrm{HFms}^{2}$, (c) LFnu, (d) HFnu, and (e) LF/HF during exercise and the comparison in control and experimental protocols. Final 5 minutes of rest (M1) and minutes of exercise: 25-30 (M2), 55-60 (M3), 85-90 (M4). *Different from M2, M3, and M4 ( $p<0.05)$; \#Different from M4 in the CP $\left(\mathrm{LF} \mathrm{ms}^{2}\right)$ and the EP $\left(\mathrm{HF}\right.$ ms $\left.{ }^{2}\right)(p<0.05)$; LF, low frequency; HF, high frequency; M, moment; ms, milliseconds; nu, normalized units.

Figures 4 and 5 present the behavior of the HRV index in the time and frequency domains, respectively, during recovery. In relation to the time domain indices, we observed moment effects in the analyzed indices and interactions between moment and protocol (SDNN and RMSSD, $p<0.001$ ). Effect of the protocol on RMSSD (ms) $(p=0.04)$ was observed, but not observed for the SDNN ( $p=0.12)$. Regarding the comparison of the SDNN (ms) index between recovery and rest, it was significantly reduced at M5, M6, M7, M8, and M9 of recovery compared to $\mathrm{M} 1$ (rest) in the CP, while it was significantly decreased at M5 compared to M1 (rest) in the EP. Regarding RMSSD (ms), it was significantly reduced at M5, M6, and M7 of recovery compared to M1 (rest) in the EP, whereas it was significantly decreased at M5, M6, M7, M8, and M9 of recovery compared to $\mathrm{M} 1$ (rest) in the CP.

In relation to the frequency domain, moment effect was observed in all indices analyzed $(p<0.001)$. Effects were observed for those indices between the protocols $\left[\mathrm{HF}\left(\mathrm{ms}^{2}\right), p=0.03\right.$; LF (nu), $p=0.03$; HF (nu), $p=0.03]$, except for the LF/HF ratio ( $p$ $=0.18)$ and $\operatorname{LF}\left(\mathrm{ms}^{2}\right)(p=0.87)$. Interactions between moment and protocol were observed for those indices [HF $\left(\mathrm{ms}^{2}\right), p=$ 0.002; LF (nu), $p=0.004$; HF (nu), $p=0.004$ ], except for the $\mathrm{LF} / \mathrm{HF}$ ratio $(p=0.62)$ and $\mathrm{LF}\left(\mathrm{ms}^{2}\right)(p=0.96)$.

The LF $\left(\mathrm{ms}^{2}\right)$ index was reduced at M5 and M6 of recovery compared to M1 (rest) in the CP while it was significantly decreased at M5 of recovery compared to M1 (rest) in the EP. HF $\left(\mathrm{ms}^{2}\right)$ was significantly reduced at M5, M6, M7, and $\mathrm{M} 8$ of recovery compared to $\mathrm{M} 1$ (rest) in the $\mathrm{CP}$, while it was significantly decreased at M5 and M6 of recovery compared to M1 (rest) in the EP. In relation to LF (nu), it was significantly increased at M5, M6, M7, M8, and M9 of recovery compared to M1 (rest) in the CP, whereas it was significantly increased at M5 of recovery compared to M1 (rest) in the EP. $\mathrm{HF}$ (nu) was significantly reduced at M5, M6, M7, M8, and M9 of recovery compared to M1 (rest) in the CP while it was significantly lower at M5 of recovery compared to M1 (rest) in the EP. LF/HF ratio was significantly higher at M5, M6, M7, M8, and M9 of recovery compared to M1 (rest) in the $\mathrm{CP}$ and significantly increased at M5 of recovery compared to $\mathrm{M} 1$ (rest) in the EP. 

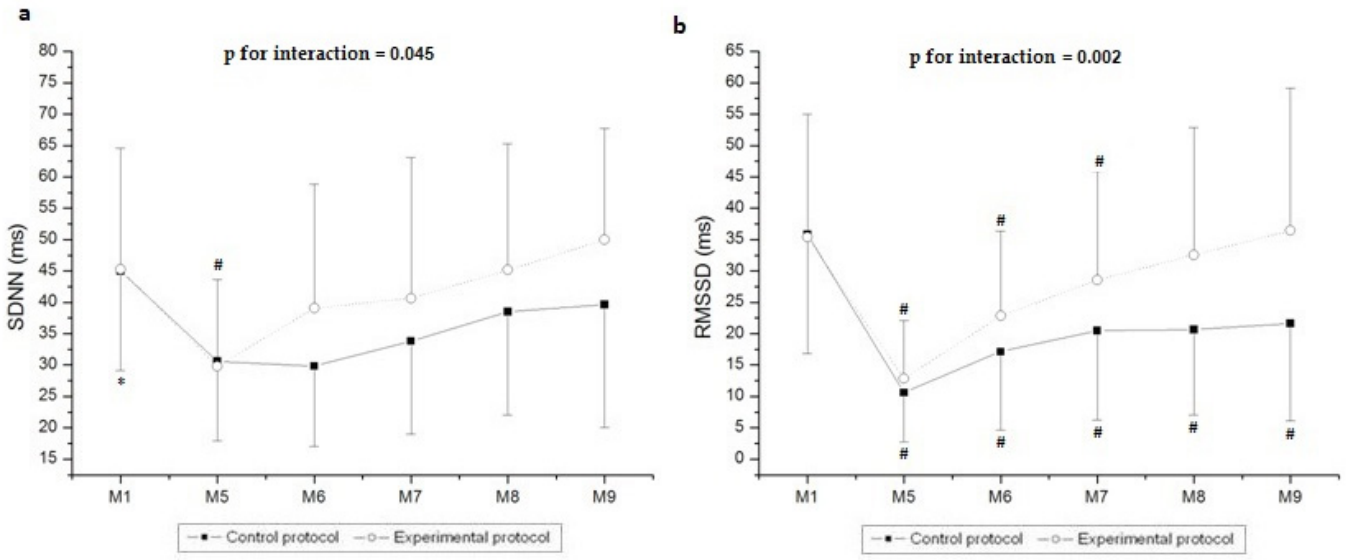

Figure 4. Values are mean \pm standard deviation. (a) SDNN and (b) RMSSD during recovery and the comparison in control and experimental protocols. Final 5 minutes of rest (M1) and minutes of recovery: 5-10 (M5), 15-20 (M6), 25-30 (M7), 40-45 (M8), 55-60 (M9). *Different from M5, M6, M7, M8, and M9 ( $p<0.05)$; \#Different from M1 $(p<0.05)$; SDNN, standard deviation of the mean of all normal RR intervals; RMSSD, square root of the mean squared differences between successive normal RR intervals; M, moment; ms, milliseconds.
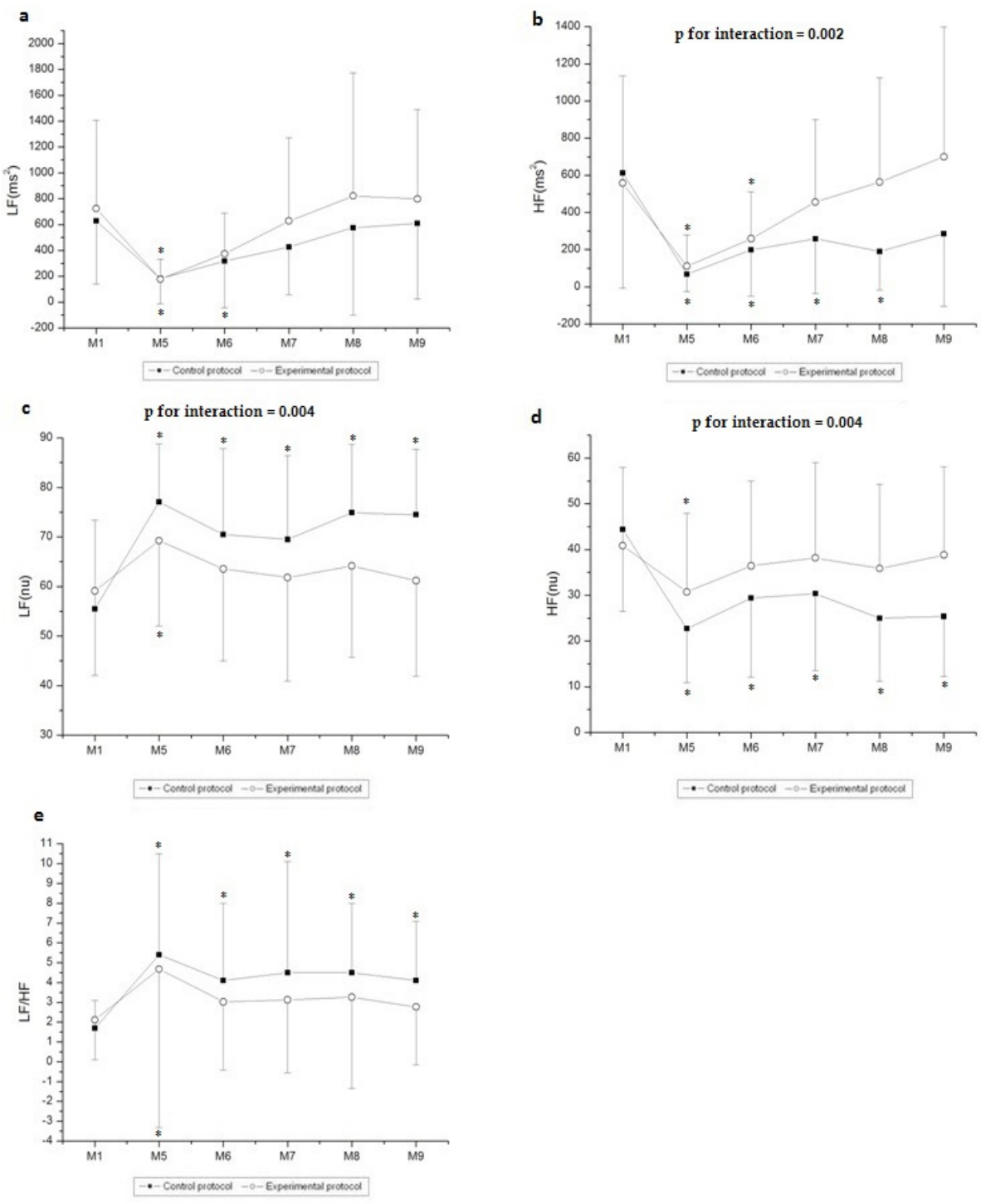

Figure 5. Values are mean \pm standard deviation. (a) $\mathrm{LFms}^{2}$, (b) HFms ${ }^{2}$, (c) LFnu, (d) HFnu, and(e) LF/HF during recovery and the comparison in control and experimental protocols. Final 5 minutes of rest (M1) and minutes of recovery: 5-10 (M5), 15-20 (M6), 25-30 (M7), 40-45 (M8), 55-60 (M9). *Different from M1 $(p<0.05)$; LF, low frequency; HF, high frequency; M, moment; ms, milliseconds; nu, normalized units. 


\section{Discussion}

The results obtained in this study show that the hydration protocol, despite having produced generally smaller changes in the HRV indices, failed to significantly influence these variables during exercise. However, water intake during the recovery period induced significant changes in cardiac autonomic modulation, promoting faster recovery of HRV indices.

It is well known that the loss of fluids through sweating, is the fundamental mechanism of heat dissipation during exercise, resulting in decreased plasma volume $(\mathrm{PV})^{20}$. An early study observed that loss of $2.1 \%$ of body weight resulted in an average reduction of 5.2\% in $\mathrm{PV}^{21}$. Despite PV not being rated in this study, we observed a loss of $2.0 \pm 0.6 \%$ of body weight when participants were not hydrated. Additionally, a study suggested that the reduction in stroke volume in participants dehydrated during exercise of moderate intensity is associated with a reduction in blood volume and an increase in HR and body temperature ${ }^{22}$. In our study, body temperature exhibited similar behavior in both protocols - which was also reported by Horswill, Stofan, Lovett and Hannasch ${ }^{23}$ - and were not observed significant differences in HR during exercise when water was administered to volunteers in comparison to the $\mathrm{CP}$.

Regarding to cardiac autonomic modulation, no significant interaction of the hydration protocol on the HRV indices analyzed was observed despite the predominance of sympathetic modulation on vagal tone during exercise. The RMSSD (ms) and HF (nu) indices, reflecting predominantly parasympathetic drive ${ }^{24}$, decreased during exercise, although they exhibited higher, but not significant values, when water was administered to volunteers in comparison to $\mathrm{CP}$. The reduction in PV resulting from the loss of body weight during exercise is responsible for reducing vagal modulation in dehydrated individuals ${ }^{25-27}$, and possibly influenced by the lower values of RMSSD (ms) and HF (nu) in the non-hydrated condition.

Reduction in global HRV is expected during exercise ${ }^{28}$ as well as increase in HR, stroke volume, cardiac output, and SBP, to supply the metabolic requirements. These mechanisms may explain the increased in LF (nu) index during exercise, that is predominantly modulated by sympathetic modulation, and also the increase in LF/HF ratio, reflecting the sympathovagal balance. ${ }^{11}$ Accordingly, the increase in the spectral indices suggests a sympathetic activation during exercise at low and moderate intensities ${ }^{29}$. Similarly, it was reported reduced SDNN (ms), RMSSD (ms) and $\mathrm{HF}$ and increased LF during exercise in individuals subjected to 8 minutes of the step test at $70 \%$ of the maximal potency ${ }^{30}$.

During exercise, as a consequence of reduced cardiac vagal modulation, the reduction of global HRV is accompanied by a decrease in absolute power $\left(\mathrm{ms}^{2}\right)$ of the spectral components $\mathrm{s}^{31}$. This behavior was also observed in the present study: $\mathrm{LF}\left(\mathrm{ms}^{2}\right)$ and $\mathrm{HF}\left(\mathrm{ms}^{2}\right)$ indices decreased during exercise compared with the rest, regardless of the administration of water. The literature indicates that both spectral indices decreased according to exercise intensity ${ }^{32}$. Therefore, we expected minimal changes to be observed in these indices due to the work load maintenance during exercise in our study.

It is important to note that during exercise other aspects, such as central command, mechanoreflex, and metaboreflex stimuli are probably more potent than hydration to influence the changes in autonomic modulation, which could also justify the absence of changes observed during exercise between protocols.

When the recovery period is analyzed, the hydration protocols promoted faster HR recovery (40 minutes post-exercise) compared with the condition in which no rehydration fluid was offered, as evidenced by the significant interaction between time and protocols on this parameter. Significant interaction between time and protocols was also observed after supplying water at the beginning and at 15,35 and 55 minutes of exercise for 16 participants $^{33}$. They found that from the tenth minute of recovery, $\mathrm{HR}$ in the poorly hydrated condition was significantly higher $(100 \pm 4 \mathrm{bpm})$ until the end of the experiment compared to well-hydrated condition $(90 \pm 4 \mathrm{bpm})$.

According to Hendrickse and Triger ${ }^{34}$, the function of volume retention by the sympathetic system is checked by the reciprocal activity of the vagal system, which promotes diuresis of salt and water. Yun, Lee, and Bazar ${ }^{35}$ suggest that hydration reduces the sympathetic drive to maintain volume and increases the parasympathetic impulse to promote diuresis. Additionally, the modulation of baroreceptors during gastric distension may be another factor that promotes reduction of sympathetic modulation due to a secondary effect of increased vagal afferent modulation ${ }^{35}$. A previous study reported that the ingestion of $500 \mathrm{~mL}$ of water caused a bradycardic response followed by an increase in cardiac vagal modulation ${ }^{36}$. These factors may have influenced the pattern of HR response observed in this study when water was administered as well as the behavior of HRV indices.

The indices that reflect the predominance of vagal modulation, RMSSD (ms), HF ( $\mathrm{ms}^{2}$ ) and HF (nu) showed a gradual increase and rapid recovery, observed in around 40 minutes when the participants were hydrated with water. In contrast, complete recovery of these indices in hypohydrated individuals was not observed. Additionally, a significant interaction between time and protocol was observed for all these indices, which suggests better recovery after exercise in the hydrated condition.

Similarly, the indices that reflect predominantly sympathetic modulation, $\mathrm{LF}\left[\mathrm{ms}^{2}\right]$ and LF [nu], also recovered better in the EP, especially the LF [nu] index which at 15 minutes post-exercise returned to the baseline. Despite the LF $\left[\mathrm{ms}^{2}\right]$ index having shown similar behavior in both protocols, the LF index [nu] revealed a significant interaction between time and protocols, suggesting faster recovery after exercise in the hydrated condition and thus sympathetic predominance in hypohydrated individuals.

Therefore, it is suggested that the process of water deficit, closely associated with the practice of physical activity resulting in $2 \%$ loss of body weight observed in the CP of this study, altered the osmotic balance and favored the predominance of post-exercise sympathetic outflow in hypohydrated individuals ${ }^{37}$.

The LF/HF ratio confirms the sympathetic predominance in hypohydrated individuals in the recovery period. Despite the lack of significant interaction between time and protocols being observed, the sympathetic-vagal balance was lower in the EP compared to $\mathrm{CP}$, with recovery of this index being observed, at 15 minutes, in the hydrated condition. Indeed, hydration can 
reduce the sympathetic-vagal ratio by reducing sympathetic modulation through modulation of cardiovascular baroreceptors $^{35}$. Accordingly, the rapid post-exercise cardiac vagal reactivation observed when $500 \mathrm{~mL}$ of water were ingested after 30 minute submaximal exercise, was caused mainly by osmotic mechanisms which, mediated by stimulation of osmosensitive structures in the gastrointestinal tract or in the circulation portal, potentially influenced cardiac vagal modulation ${ }^{6}$.

In this study, the hypohydrated state also reduced overall HRV after exercise. Significant interaction was observed between moments and protocols for SDNN [ms], suggesting better post-exercise recovery of these indices in the hydrated condition, and given that at 15 minute post-exercise the return of this index to baseline was observed. This response confirms the influence of hydration status on post-exercise cardiac autonomic stability.

This study has some limitations. The minimum interval between the execution of the CP and the EP was adhered to; however, some collections were completed over a period longer than a week, which may hinder the interpretation of the variables studied. Another important aspect refers to the use of supine rest and recovery conditions, considering that this exercise was performed in the upright position. Although we chose to compare rest and exercise in different positions, we believed that the modifications produced in the parameters during exercise were not influenced by the postural change. However, in addition to being more tolerable for the volunteer, the choice of the supine position during the recovery period has not impaired the results because the parameters were compared to a baseline, with participants in the same position. Finally, the absence of randomization between protocols can be influenced the results, however, for the dynamic correct of trial was necessary to accomplish firstly the CP to determine the loss of body mass and based on these data suggest the amount of water to be consumed in the hydration protocol.

However, the results of this study reinforce the importance of hydration when performing physical exercise to promote a better response in cardiac autonomic modulation. In sports scope, a faster recovery of the autonomic modulation indicates that the autonomic nervous system of the individual quickly is ready to receive a new stimulus, while in the clinical scope, a better recovery of the autonomic modulation may be important to prevent or reduce cardiovascular events after exercise such as cardiac arrhythmias, decreased or increased blood pressure, pulmonary hyperventilation increasing body $\mathrm{pH}$ and an excessive production of metabolites ${ }^{38.39}$. As a future perspective, investigating the influence of water intake over exercise and recovery on sodium concentration and PV can bring better knowledge of this hydration protocol.

\section{Conclusion}

In young participants, hydration with small amounts of water ingested throughout exercise and recovery did not change autonomic regulation during the exercise, but accelerated autonomic recovery measured by HRV indices in the time and frequency domains.

\section{References}

1. Hernandez AJ, Nahas RM. Dietary changes, water replacement, food supplements and drugs: evidence of ergogenic action and potential health risks. Rev Bras Med Esporte. 2009;15: 3-12.

2. Moreno IL, Pastre CM, Papoti M, Vanderlei LCM. Efeitos da reposição hidroeletrolítica sobre parâmetros cardiorrespiratórios em exercício e recuperação. Motriz. 2012; 18:165-175.

3. Rodriguez NR, DiMarco NM, Langley S. Nutrition and athletic performance. Med Sci Sports Exerc. 2009; 41: 709-731.

4. Sawka MN, Burke LM, Eichner ER, Maughan RJ, Montain SJ, Stachenfeld NS. American college of sports medicine position stand. Exercise and fluid replacement. Med Sci Sports Exerc. 2007; 39: 377-390.

5. Montain SJ. Hydration recommendations for sport 2008. Curr Sports Med Reports. 2008; 7;187-192.

6. Vianna LC, Oliveira RB, Silva BM, Ricardo DR, Araújo CG. Water intake accelerates post-exercise cardiac vagal reactivation in humans. Eu J Appl Physiol. 2008; 102: 283-288.

7. Moreno IL, Vanderlei LC, Pastre CM, Vanderlei FM, Abreu LC, Ferreira C. Cardiorespiratory effects of water ingestion during and after exercise. Int Arch Med. 2013; 6: 35.

8. Peçanha T, Paula-Ribeiro M, Campana-Rezende E, Bartels R, Marins JC, de Lima JR. Water intake accelerates parasympathetic reactivation after high-intensity exercise. International J Sport Nutr Exerc Metab. 2014; 24: 489-496.

9. Naugle KM, Riley JL. Self-reported physical activity predicts pain inhibitory and facilitatory function. Med Sci Sports Exerc. 2014; 46:622-629.

10. Moreno IL, Pastre CM, Ferreira C, Abreu LC, Valenti VE, Vanderrlei LCM. Effects of an isotonic beverage on autonomic regulation during and after exercise. J Society Sports Nutr. 2013; 10:2-11.

11. Vanderlei FM, Moreno IL, Vanderlei LCM, Pastre CM, Abreu LC, Ferreira C. Comparison of the effects of hydration with water or isotonic solution on the recovery of cardiac autonomic modulation. Int J Sport Nutr Exerc Metab. 2015; 25:145-153.

12. Shah BN. On the 50th anniversary of the first description of a multistage exercise treadmill test: re-visiting the birth of the 'Bruce protocol'. Heart. 2013; 99:1793-1794.

13. Costill DL, Sparks KE. Rapid fluid replacement following thermal dehydration. J Appl Physiol. 1973; 34:299-303.

14. Von Duvillard SP, Braun WA, Markofski M, Beneke R, Leithäuser R. Fluids and hydration in prolonged endurance performance. Nutrition. 2004; 20:651-656.

15. Armstrong LE, Pumerantz AC, Fiala KA, Roti MW, Kavouras SA, Casa DJ, Maresh CM. Human hydration indices: acute and longitudinal reference values. Int J Sport Nutr Exerc Metab. 2010; 20:145-153.

16. Task Force of the European Society of Cardiology of the North American Society of pacing electrophysiology. Heart rate variability standards of measurement, physiological interpretation and clinical use. Circulation. 1996; 93: 1043-1065.

17. Godoy MF, Takakura IT, Correa PR. The relevance of nonlinear dynamic analysis (Chaos Theory) to predict morbidity and mortality in patients undergoing surgical myocardial revascularization. Arq Ciências Saúde. 2005; 12: 167-171. 
18. Corrêa PR, Catai AM, Takakura IT, Machado MN, Godoy MF. Heart rate variability and pulmonary infections after myocardial revascularization. Arq Bras Cardiol. 2010; 95: 448-456.

19. Tarvainen MP, Niskanen JP, Lipponen JA, Ranta-aho PO, Karjalainen PA. Kubios HRV - A software for advanced heart rate variability analysis. In: 4th European Conference os the International Federation for Medical and Biological Engineering, Sloten JV, Verdonck P, Nyssen M, Haueisen J, editors. 2008, pp. 1022-1025.

20. Sawka MN, Young AJ. Physiological systems and their responses to conditions of heat and cold. In: Tipton CM, Sawka MN, Tate CA, Terjung RL, editors. ACSM's Advanced Exercises Physiology. Baltimore: Lippincott, Williams \& Wilkins; 2006. p. 535-63.

21. Maughan RJ, Leiper JB, Shirreffs SM. Restoration of fluid balance after exercise induced dehydration: effects of food and fluid intake. Eur J Appl Physiol. 1996; 73: 317-325.

22. González-Alonso J, Mora-Rodríguez R, Coyle EF. Stroke volume during exercise: interaction of environment and hydration. Am J Physiol: Heart Circ Physiol. 2000; 278:H321-H330.

23. Horswill CA, Stofan JR, Lovett SC, Hannasch C. Core temperature and metabolic responses after carbohydrate intake during exercise at 30 degrees C. J Athletic Training. 2008; 43:585-591.

24. Vanderlei LCM, Pastre CM, Hoshi RA, Carvalho TD, Godoy MF. Basic notions of heart rate variability and its clinical applicability. Rev Bras Cirurgia Cardiovascular. 2009; 24:205-217.

25. González-Alonso J, Mora-Rodríguez R, Below PR, Coyle EF. Dehydration markedly impairs cardiovascular function in hyperthermic endurance athletes during exercise. J Appl Physiol. 1997; 82: 1229-1236.

26. Crandall CG, Zhang R, Levine BD. Effects of whole body heating on dynamic baroreflex regulation of heart rate in humans. Am J Physiol: Heart Circ Physiol. 2000; 279:H2486-2492.

27. Charkoudian N, Halliwill JR, Morgan BJ, Eisenach JH, Joyner MJ. Influences of hydration on postexercise cardiovascular control in humans. J Physiol. 2003; 552, 635-44.

28. Alonso DO, Forjaz CLM, Rezende LO, Braga AM, Barretto AC, Negrão CE, Rondon MU. Heart rate response and its variability during different phases of maximal graded exercise. Arq Bras Cardiol. 1998; 71:787-792.

29. Mendonca GV, Fernhall B, Heffernan KS, Pereira FD. Spectral methods of heart rate variability analysis during dynamic exercise. Clin Auton Res. 2009; 19:237-245.

30. Javorka M, Zila I, Balhárek T, Javorka K. Heart rate recovery after exercise: relations to heart rate variability and complexity. Braz J Med Biol Res. 2002; 35:991-1000.

31. Perini R, Veicsteinas A. Heart rate variability and autonomic activity at rest and during exercise in various physiological conditions. Eur J Appl Physiol. 2003; 90:317-325.
32. Sandercock GRH, Brodie DA. The use of heart rate variability measures to assess autonomic control during exercise. Scand J Med Sci Sports. 2006; 16:302-313.

33. Saat M, Tochihara Y, Hashiguchi N, Sirisinghe RG, Fujita M, Chou CM. Effects of exercise in the heat on thermoregulation of Japanese and Malaysian males. J Physiol Anthropol Appl Human Sci. 2005; 24:267-275.

34. Hendrickse MT, Triger DR. Vagal dysfunction and impaired urinary sodium and water excretion in cirrhosis. Am J Gastroenterol. 1994; 89:750-757.

35. Yun AJ, Lee PY, Bazar KA. Clinical benefits of hydration and volume expansion in a wide range of illnesses may be attributable to reduction of sympatho-vagal ratio. Med Hypotheses. 2005; 64:646-650.

36. Routledge HC, Chowdhary S, Coote JH, Townend JN. Cardiac vagal response to water ingestion in normal human subjects. Clin Sci. 2002; 103:157-162.

37. Scrogin KE, Grygielko ET, Brooks VL. Osmolality: a physiological long-term regulator of lumbar sympathetic nerve activity and arterial pressure. Am J Physiol. 1999; 276: R1579-R1586.

38. Brum PC, Forjaz CLM, Tinucci T, Negrão CE. Adaptações agudas e crônicas do exercício físico no sistema cardiovascular. Rev Paulista Educação Física. 2004; 18: 21-31.

39. Guyton A, Hall J. Tratado de Fisiologia Médica (11 a ed.). Brasil: Elsevier; 2006.

\section{Funding}

This study was support by the Foundation for Research Support of São Paulo State (FAPESP-Proc. 2011/10887-7).

\section{Corresponding Author}

Isadora Lessa Moreno

Department of Medicine

Rua Napoleão de Barros, 715, Térreo, São Paulo, SP, Brasil.

Email: moreno.isadoralessa@gmail.com

Manuscript submitted: October 15, 2015

Manuscript accepted: April 10, 2016

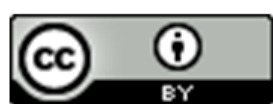

Motriz. The Journal of Physical Education. UNESP. Rio Claro, SP, Brazil - eISSN: 1980-6574 - under a license Creative Commons - Version 3.0 